\title{
Unbalanced GTS Assignment Mechanism based on Delay Constraint in IEEE802.15.4
}

\author{
Fengjun Shang, Qiang Fu and Ran Mao \\ College of Computer Science and Technology, Chongqing University of Posts \\ and Telecommunications, Chongqing 400065, China
}

\begin{abstract}
This article studied the GTS allocation mechanism of the IEEE802.15.4 protocol. Firstly, it discusses the existing deficiencies in the standard and studies the advantages and disadvantages of other improved algorithm. A mechanism is proposed in this paper breaking the original standard fixed GTS timeslot number firstly and making the time slot allocation more flexible. Secondly, it considers the demand that some nodes have special request for time delay and bandwidth, it analysis the constraints by using network calculus theory and tests nodes whether meet the allocation principle. Finally, it allocates the resources for nodes which meet the demand using their priority. Experiments show that this improved GTS allocation mechanism raises the time slot bandwidth utilization rate, satisfy the special requirements for some nodes, reduce the packet delay.
\end{abstract}

Keywords: Wireless Sensor Network, IEEE802.15.4, GTS allocation, network calculus

\section{Introduction}

Wireless sensor network is a new kind of data monitoring network, which is developed with the development of wireless communication technology, embedded computing technology, sensor technology and network technology. It is considered as one of the most important technologies in twenty-first Century, and has broad application prospect. ZigBee technology is a new kind of low cost communication protocol for wireless sensor networks. It is a low power IEEE802.15.4 protocol based on standard [1]. It is the first choice for wireless sensor networks. As one of the important key layers in wireless sensor network architecture, Medium Access Control (MAC) is one of the key nodes in wireless sensor networks, which is one of the key points of wireless sensor networks [2].

\section{Unbalanced GTS Assignment Mechanism Based on Delay Constraint}

In the IEEE802.15.4 standard, in order to distribute the GTS in the non-competition period (CFP), the active part is divided into 16 time slots, a GTS consists of a number of integer slots, the optional CFP can accommodate up to 7 GTS. A device that wants to use GTS to send or receive data packets requires that a GTS request packet is sent to the PAN center node in a competitive period (CAP). After calculating all the available resources, the PAN node sends a list of GTS users with GTS information to all nodes in the next super frame.

In this paper, it will resolve that the GTS bandwidth is not fully utilized when the guaranteed bandwidth is higher than the request bandwidth. When a device requests a guaranteed bandwidth, it is assigned a GTS containing multiple slots. The duration of a time slot is composed of a super frame number (SO) and a beacon (BO). In a real-time 
wireless sensor network, nodes generate a large amount of burst data and need to be sent immediately to the PAN coordinator. This leads to a phenomenon that the preconfigured GTS mechanism allocates time slots for a node that has no data packets to transmit, and the GTS bandwidth utilization will fall [3]. If the period of the data is much lower than the time slot, then the GTS bandwidth will be wasted. In addition, in different time periods, the GTS request packet is not supported. GTS time depends on the super frame structure and network configuration. TuanNam Le proposes UGAS Unblanced [4] (GTS Allocation Scheme) algorithm, but the algorithm will be the original algorithm in each time slot is divided into 8 . Therefore, this paper proposes a new efficient GTS allocation mechanism, called the unbalanced GTS distribution mechanism. In contrast to the IEEE802.15.4 standard GTS mechanism, we propose that the mechanism is not equal to the slot using the same time slot. The standard PAN node for the request of the GTS node is the first service, that is, as long as there is a surplus of time slots will be allocated to the node, and we put forward the mechanism is the request packet priority, and then allocated. Different from the UGAS algorithm, the proposed algorithm is divided into two parts in the original standard, and the maximum allocation equipment is no longer limited to 7 . The mechanism is efficient in bandwidth allocation, and it is not necessary to develop complex protocols.

\subsection{Dividing Super Frame Structure}

Assuming that the packet length is higher than the 144bits, then in the CFP, the longest time of the successfully transmitted to one of the longest data packets is $t_{\max \text { Packet }}$, and the calculation formula is as follows:

$$
t_{\max \text { Packet }}=t_{\text {packet }}+t_{A C K}+t_{\text {turnaround }}+t_{L I F S}=328(\text { symbol })
$$

The shortest length of CAP is aMinCAPLength (440symbol), that is, $7.04 \mathrm{~ms}$, IEEE802.15.4. If a node has only one packet to be transmitted, the time of a time slot should be sufficient to transmit data packets.

$$
\begin{aligned}
& \text { aBaseSuperFrameDuration } \times 2^{\text {SO }}-\text { symbol }_{\text {Beacon }} \\
& \geq t_{\max \text { packet }} \times \text { num_min } i-\text { slot }
\end{aligned}
$$

It can be drawn from the formula 2.2:

$$
n u m_{-} \min i-\operatorname{slot} \leq \operatorname{ceil}\left(\frac{960 * 2^{S O}-\text { symbol }_{\text {Beacon }}}{t_{\text {max }_{-} \text {packet }}}\right)
$$

Figure 2.1 is the standard super frame format and the new super frame format:

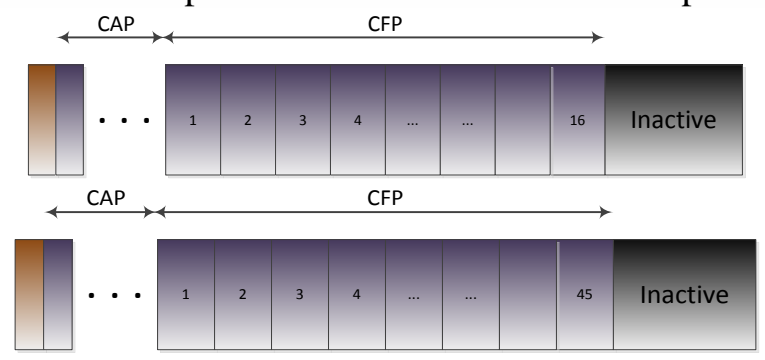

Figure 2.1. Standard Frame Format, New Super Frame Format

From figure 2.1, we can find that the time slot allocation mechanism based on the original standard is 16 time slots, which makes the time slot allocation more flexible and effective. In the IEEE802.15.4 standard, the coordinator can allocate up to 7 GTS, each GTS is composed of one or more slots. Using the new super frame format, you can increase the number of GTS allocation, so that more than 7 devices can also request GTS resources. 


\subsection{Model Research and Constraint Analysis}

Network calculus theory is a deterministic model in communication network, which can analyze the security mechanism and the application of continuous data stream transmission [5]. Using this theory, we can help us to better understand the basic service information in the network, the window flow control, the timing, the size of the cache area or the transmission delay.

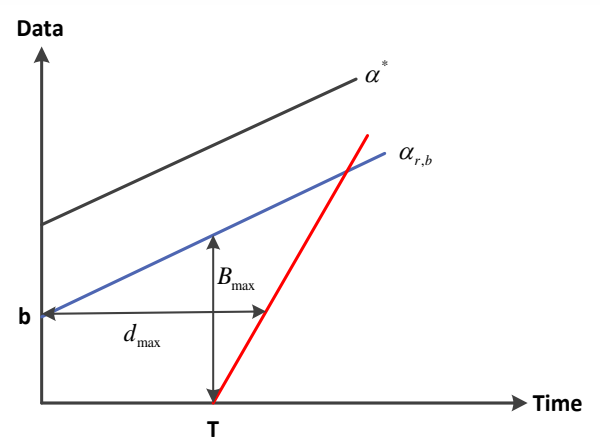

\section{Figure 2.2. Network Calculus Model}

Figure 2.2 is the network calculus data flow model, which shows the relationship between the service curve and the arrival rate curve of the data stream, which is the important factor of the QoS in the integrated service network. Assuming that the data flow to the function is $\mathrm{R}(\mathrm{T})$, the arrival curve is $\alpha(t)$, where $0 \leq s \leq t$, $R(t)-R(s) \leq \alpha(t-s)$. Delay bounds $d_{\max }$ shows the arrival curves and service curves. The calculation formula is as follows:

$$
d_{\max }=T+b / R
$$

In the above formula, $T$ represents the maximum time delay for a service to wait for a service; $b$ is a burst constraint; $C$ is the service rate specified in the IEEE802.15.4 standard 250kbps. The buffer boundary is $\alpha_{r, b}$ and Time maximum vertical distance of the two curves is $B_{\max }$ and the calculation formula is as follows:

$$
B_{\text {max }}=b+r * t
$$

Where $\mathrm{R}$ is the average arrival rate of the data stream, $r \leqslant R$. Otherwise the value of the buffer boundary becomes infinite. In addition, it can be concluded that the buffer boundary value is determined by the maximum horizontal distance, and the calculation formula is as follows:

$$
b_{\max }=b+r * T
$$

We first calculate the time required to transmit a packet in a CFP, and the process of transmitting data packets in a time slot is shown in figure 2.3 [6].

GTS slot

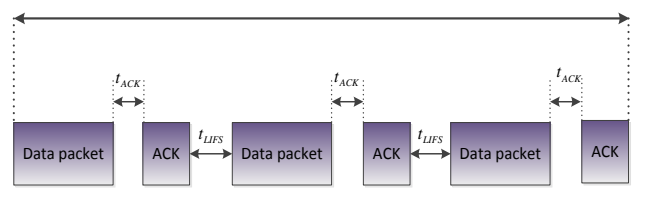

Figure 2.3. GTS Packet Transmission Process

The size of each time slot $t_{s}$ in the IEEE802.15.4 standard is as follows: 


$$
\begin{gathered}
t_{s}=\frac{S D}{16}=\frac{\text { aBaseSuperFrameDuration } \times 2^{\text {SO }}}{16} \\
=\text { aBaseSuperFrameDuration } \times 2^{\text {so- } 4} \\
\text { aBaseSuperFrameDuration }=B_{s} * S_{a}(\text { symbol })
\end{gathered}
$$

Where $\mathrm{SO}$ is a super frame number, $\mathrm{B}_{\mathrm{s}}$ is 60 symbol, $\mathrm{S}_{\mathrm{a}}$ is the number of active time slots, the default value is 16 , then you can calculate the symbol value of each time slot Symbol_slot. Formula is as follows:

$$
\text { Symbol_slot }=B_{s} * 2^{\text {SO }}
$$

Assuming a GTS contains n slots, then the throughput of this GTS tGTS is calculated as follows:

$$
t_{G T S}=n * \text { Symbol_slot }
$$

The shortest length of CAP is (440symbol), that is $7.04 \mathrm{~ms}$; then we can calculate the maximum number of time slots $\mathrm{Num}_{\text {slots }_{-} \text {of }} \mathrm{CFP}_{\text {in }}$ in the standard CFP:

$$
\text { Num }_{\text {slot__of_CFP }}=15-\text { ceil }\left(\frac{440}{\text { Symbol_slot }}\right)
$$

Assuming that the packet length is higher than the 144bits, then in the CFP, $t_{\max \text { Packet }}$ is successfully transmitted one of the longest data packets, and the calculation formula is as follows:

$$
t_{\text {max Packet }}=t_{\text {packet }}+t_{A C K}+t_{\text {turnaround }}+t_{\text {LIFS }}=328(\text { symbol })
$$

Among them, the maximum load of the physical layer specified is 127 bytes in IEEE802.15.4 [7], a recognized frame is 11 bytes, which the value is 12 symbol.

In addition to modifying the super frame structure, this paper also improves the MLME-GTS.request frame structure of the sensor to the coordinator node applying for GTS [5]. The modified frame format is shown in figure 2.4.

\begin{tabular}{|c|c|c|c|c|}
\hline$\ldots$ & Octets:2 & Variable & Variable & $\ldots$ \\
\hline$\ldots$ & GTS direction & GTS field & $\begin{array}{c}\text { Pending } \\
\text { address }\end{array}$ & $\ldots$ \\
\hline
\end{tabular}

\begin{tabular}{|c|c|c|c|}
\cline { 2 - 4 } \multicolumn{1}{c|}{} & Octets:1 & $0 / 1$ & Variable \\
\cline { 2 - 4 } & $\begin{array}{c}\text { GTS } \\
\text { specification }\end{array}$ & GTS direction & GTS list \\
\hline Bits:0-3 & 4 & 5 & $6-7$ \\
\hline GTS length & GTS direction & $\begin{array}{c}\text { Characteristi } \\
\text { cs }\end{array}$ & $\begin{array}{c}\text { Delay_requir } \\
\text { e }\end{array}$ \\
\hline
\end{tabular}

\section{Figure 2.4. Modified Request Frame Format}

The standard reserves $6^{\text {th }}$ and $7^{\text {th }}$ bit, so this paper will requirement each sensor node on the delay value on the reservation. In this paper, the value of delay from 100 to 600 in random uniform distribution, set as follows:

The burst data stream size can be obtained by using the MAC layer buffer size. Then we assume that a super frame internal coordinator node should be allocated to the number of slots $n_{\text {slot }}$ for the application resource. The calculation formula is as follows: 


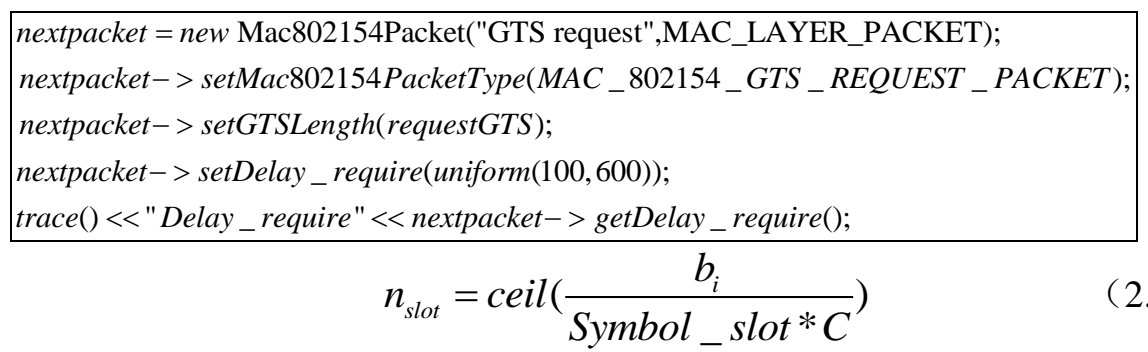

Through the research of network calculus theory, we can guarantee the QoS of all nodes in the whole network. According to the above formula, we can get the QoS constraint conditions $d_{i, \max }$ and $b_{i, \max }$ of sensor node data flow. Where $d_{\max }$ is the request of the node in the GTS request packet and $B_{\max }$ is the limit of the maximum value of the node buffer, and the calculation formula is as follows:

$$
\begin{gathered}
d_{i, \text { max }}=\frac{b_{i} * B I}{n_{\text {slot }}\left(t_{\max \text { Packet }} * C\right)}+\left(B I-n * t_{s}\right) \\
d_{i, \text { max }} \leq d_{\max } \\
b_{i, \text { max }}=b_{i}+\text { ceil }\left(\frac{b_{i} * t_{\text {packet }} * 2}{\text { Symbol_slot }}\right) * t_{s} * r_{i} \\
b_{i, \text { max }} \leq B_{\max } \\
R_{i}=\text { ceil }\left(\frac{b_{i} * t_{\text {packet }} * 2}{\text { Symbol_slot }}\right) * \frac{t_{\text {packet }} * C}{B I}
\end{gathered}
$$

When the node of the request GTS is satisfied with the formula 2.15 and 2.17, the coordinator receives the request, otherwise, the coordinator node will refuse their request.

\subsection{GTS Allocation Mechanism}

In the IEEE802.15.4 standard, the coordinator of the GTS resource is used by the FCFS algorithm. Coordinator node does not consider other factors, as long as there is a node to apply for resources, and the current resources to meet the needs of the case, then the coordinator to give the device resources. This situation is easy to cause the loss of bandwidth resources. If the application resource node needs to send the few packet, and the delay did not request. And then it will waste a large majority of time slot resources. But it will be unfavourable for the device requesting delay limit [8]. Therefore, in this paper, we abandon the FCFS algorithm in the resource scheduling, and learn from the GTS packet priority based on node request.

In order to verify the validity of the proposed algorithm, we assume that the SO is 4 , the time slot number in CFP is 8 , and each GTS has 2 slots. The network has 8 devices, which have 8 sets of data stream needs to be sent. They are $70 \mathrm{~ms}, 100 \mathrm{~ms}, 130 \mathrm{~ms}$, $210 \mathrm{~ms}, 320 \mathrm{~ms}, 400 \mathrm{~ms}, 550 \mathrm{~ms}$, and $600 \mathrm{~ms}$ respectively. Their buffers are $360 \mathrm{bytes}$, 500bytes, 120 bytes, 230 bytes, 70 bytes, 450 bytes, 870 bytes, and 90 bytes respectively.

Figure 2.5 is the allocation of resources using the GTS distribution mechanism for the IEEE802.15.4 standard. Since each GTS takes up two slots, the graph has only 4 GTS resources, namely $(1,2),(3,4),(5,6),(7,8)$, therefore, up to a maximum of 4 devices. From the figures, the distribution of the GTS for each device is not fully utilized, and the remaining time slots can only be wasted, not shared and recycled. Figure 2.6 is the method of UGAS distribution GTS, because the algorithm provides a time when the arrival of the beacon frame, the coordinator can give up to 7 devices to allocate resources. Moreover only 7 of the data flow in 8 data streams can use GTS slot. Because UGAS is divided into 4 mini-slot, the coordinator can adaptively allocate time slots according to the traffic flow, and it is more flexible than the standard algorithm, 
and it can also improve the GTS bandwidth utilization. The allocation mechanism of this paper is not limited to the number of devices, so it can meet the requirements of 8 devices simultaneously, GTS, and UGAS. Compared with UGAS, it improved the efficiency of GTS bandwidth, as shown in figure 2.7.

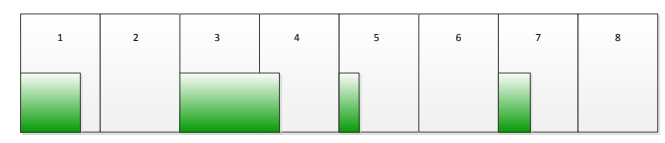

Figure 2.5. IEEE802.15.4 GTS Allocation Mechanism

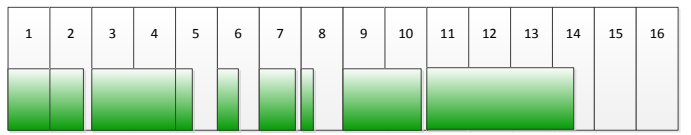

Figure 2.6. UGAS Allocation Mechanism

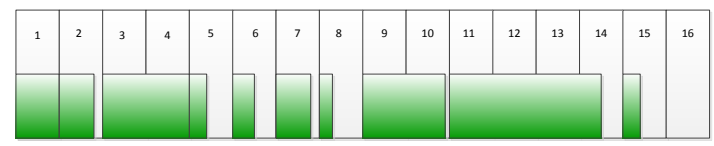

Figure 2.7. The Allocation Mechanism Proposed

\section{Simulation and Results Analysis}

In this paper, we use OMNet++ tool [9] to simulate the algorithm and establish a small star network topology, which is arranged with two scenes of sparse data flow and dense data stream. Among them, the scene 1 is a sparse data stream, 1 coordinator and 10 sensor nodes are arranged. The arrival rate of the 10 data streams is 4, 6, 8, 10, 12, $14,16,18,20,25 \mathrm{kbps}$. Scene 2 is dense data stream, the 1 coordinator node and 12 sensor terminal nodes are arranged. The arrival rate of the 10 data streams is $20,25,30$, $36,40,42,50,55,60,70 \mathrm{kbps}$. BO is 14 , Delay_require_uniform $\in(100,600)$. We measure the performance of the proposed algorithm in the case of fixed data arrival rate using the bandwidth utilization of CFP slot. We define the bandwidth utilization of time slot $U_{C F P}$ as a GTS bandwidth utilization can be expressed as follows [10]:

$$
\begin{gathered}
U_{G T S}=t / n * t_{s} \\
t=\frac{r * B I}{C} \\
(n-1) * t_{s}<t \leq n * t_{s}
\end{gathered}
$$

The bandwidth utilization of the formula 3.2 can be expressed as follows:

$$
U_{C F P}=\frac{B I * \sum_{i=1}^{m} r_{i}}{\left(\text { symbol }_{C F P}-\text { symbol }_{\text {Beacon }}-\text { symbol }_{C A P}\right) * C}
$$

Figure 3.1 shows the IEEE802.15.4 protocol, the UGAS mechanism, and the comparison of the bandwidth utilization of the proposed mechanism in the intensive data flow situation. From the figure, the proposed mechanism, higher than $7 \%$ of bandwidth utilization is used comparing with the UGAS algorithm, higher than $13 \%$ of bandwidth utilization is used comparing with the standard algorithm. The proposed mechanism based on the time and the value of SO dividing the time will lower bandwidth utilization. Compared with the UGAS algorithm, the proposed algorithm can break the constraint of the number of GTS, so the UGAS can use the data stream with a maximum of 7, and the bandwidth utilization is also decreased. With the increasing of 
SO, the data stream is constant, and the beacon interval is increasing, so the bandwidth utilization will show a downward trend.

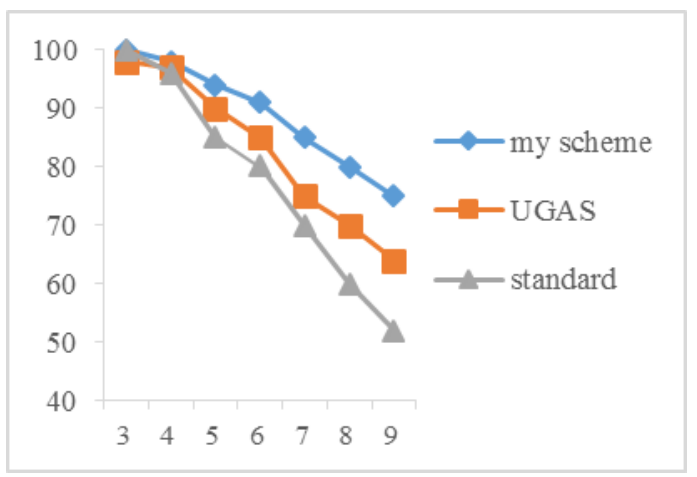

\section{Figure 3.1. Bandwidth Utilization Contrast in Intensive Data Flow}

Figure 3.2 shows the delay in the case of intensive data flow among IEEE802.15.4 protocol, the UGAS mechanism, and he proposed algorithm. It can be seen from the graph, since the proposed algorithm and UGAS algorithm both consider the node to delay, and the delay is lower than the standard, the delay between the proposed mechanism is maintained at UGAS, the delay between the $0.1 \sim 0.25$ mechanism is maintained at $0.15 \sim 0.25$, and the delay between the standard algorithm is $0.14 \sim 0.3$. When $\mathrm{SO}$ is in a certain range, the proposed mechanism can effectively control the delay boundary value, and when the $\mathrm{SO}$ is increasing, the beacon interval increases with the increase of SO, which presents exponential growth trend, so the delay will be increased.

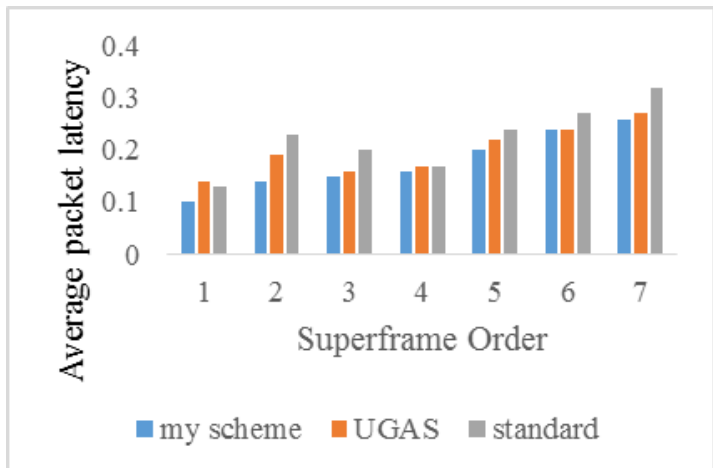

Figure 3.2. Delay Contrast in Intensive Data Flow

Figure 3.3 shows the bandwidth utilization in the case of sparse data streams among IEEE802.15.4 protocol, the UGAS mechanism, and the proposed algorithm. As can be seen from the graph, the GTS allocation algorithm in the IEEE802.15.4 allocation algorithm in bandwidth utilization is significantly lower than the other two algorithms, which is due to the optimization of the allocation algorithm, for all applications of GTS sensor nodes, regardless of the number of its data flow, the allocation of time slots are the same. UGAS algorithm although are much higher than that of the standard allocation algorithm, but due to its limited number of GTS allocation, making redundant time slots are not in use; and using the greedy algorithm to allocate the GTS, due to unbalanced slot allocation mechanism is UGAS. Therefore, when the coordinator node is assigned a time slot, the slot a multiple GTS will be preferentially allocated out, then the rest of the small number of time slots is difficult to meet the request. Therefore, with the increase of SO, the improved mechanism proposed in this paper is more than 
IEEE802.15.4 standard and UGAS. As shown in Figure 3.3, when the SO is 9, the active cycle will become longer, then the CFP stage will be long, due to the limited data flow size, will lead to a decrease in the total time slot bandwidth utilization.

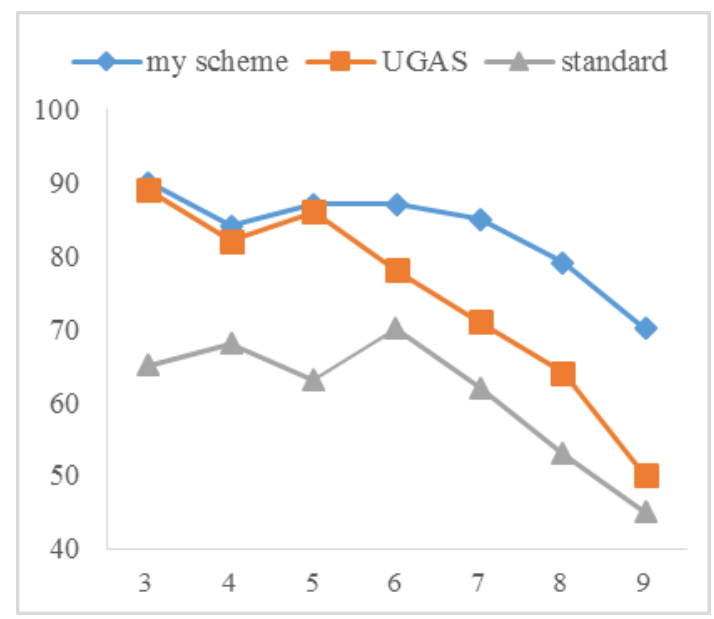

Figure3.3 Bandwidth Utilization Contrast in Sparse Data Flow

Figure 3.4 shows the delay in the case of sparse data flow among the IEEE802.15.4 protocol, the UGAS mechanism, and the proposed algorithm. It can be seen from the graph, since the proposed algorithm and UGAS algorithm both consider the node to delay, and the delay is lower than the standard, the delay between the proposed mechanism is maintained at UGAS, the delay between the 0.13 0.25 mechanism is maintained at 0.15 0.3, and the delay between the standard algorithm is $0.14 \sim 0.35$. When SO is in a certain range, the proposed mechanism can effectively control the delay boundary, and when the SO is increasing, the beacon interval $t_{s}$ increases with the increase of SO, which presents exponential growth trend, so the delay will be increased.

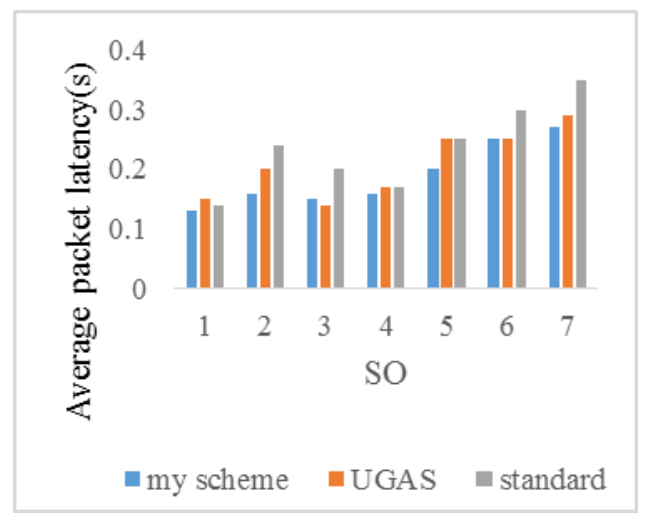

Figure 3.4. Delay Contrast in Sparse Data Flow

\section{Conclusions}

With the in-depth study of wireless sensor networks, more and more scholars begin to study the IEEE802.15 standard for wireless sensor networks to provide MAC layer and physical layer services. In this paper, we improve the distribution mechanism of GTS in the standard, and put forward the mechanism of unbalanced GTS allocation of QoS. The main research results are as follows: 
(1) GTS distribution in IEEE802.15.4 standard is used to first service and fixed time slot allocation mechanism, without the full use of time slot bandwidth, and without considering the special requirements for the delay and bandwidth of the node, and Tuan Le UGAS proposed Nam mechanism in the time slot allocation problem. Therefore, this paper takes into account the different bandwidth requirements of each data stream, the slot is divided into a number of mini-slot.

(2) Considering the different requirements of the data stream to the delay, this paper can meet the demand of data stream by setting time delay value in the GTS request. By calculating the maximum time delay value and the buffer boundary value to the node QoS to monitor, if satisfied, then accept the request of the node, otherwise refused.

(3) It is different from the standard GTS distribution mechanism, when receiving the device request GTS frame, and no immediately to the node distribution, but the first test whether it satisfies QoS. According to the equipment of the delay and priority order, if the remaining time slot enough use, it will be distributed.

\section{Acknowledgements}

The author would like to thank the Chongqing Natural Science Foundation under Grant No. cstc2012jjA40038 and the Chongqing Basic and Frontier Research Project under Grant NO.cstc2013jcyjA40023, cstc2014kjrc-qnrc40002. The work presented in this paper was supported in part by the Ministry of Industry and Information Technology of the Peoples Republic of China for the special funds of Development of the Internet of things (2012-583).

\section{References}

[1] "IEEE 802.15.4, Part 15.4: Wireless Medium Access Control (MAC) and Physical Layer (PHY) Specifications for Low-Rate Wireless Personal Area Networks (LR-WPANs)", revision of IEEE Std 802, (2003).

[2] L. Yanxiao, Z. Yueling, G. Hua and L. Dongyang, "Novel low energy consumption MAC protocol for the wireless sensor networks", Journal of Xidian University, vol. 39, no. 1, (2012), pp. 168-171.

[3] L. Xiaoji, C. Chen, Q. Hongbing and M. Wei, "Adaptive guaranteed time slots allocation policy in wireless sensor networks with mobility awareness", Journal of Communication, vol. 31, no. 10, (2010), pp. 212-220.

[4] L. Namtuan, C. Sunwoong and J. Yeongmin, "New QOS resource allocation scheme using GTS for WPANS", Wireless Personal Communications, vol. 67, no. 1, (2012), pp. 25-45.

[5] X. Qi, W. Lei and H. Jun, "GTS scheduling algorithm optimization based on IEEE802.15.4", Computer Engineering and Design, vol. 32, no. 11, (2011), pp. 3642-3645.

[6] Y. Ding and S. H. Hong, "CFP Scheduling for Real-Time Service and Energy Efficiency in the Industrial Applications of IEEE 802.15.4", Journal of Communications and Networks, vol. 15, no. 1, (2013), pp. 87-101.

[7] X. Weili, T. Mengna and X. Baoguo, "Research of MAC Protocol based on OPNET in Wireless Sensor Networks", Journal of Transduction Technology, vol. 23, no. 1, (2010), pp. 139-143.

[8] I. A. Anbagi, M. E. Kantarci and H. T. Mouftah, "Delay-Aware Medium Access Schemes for WSNBased Partial Discharge Measurement", IEEE Transactions on Instrumentation and Measurement, vol. 63, no. 12, (2014), pp. 3045-3057.

[9] L. Juan, "Wireless sensor network principle and OMNeT++ implementation", Changsha: Hunan University press, (2011).

[10] B. Shrestha, E. Hossain and S. Camorlinga, "IEEE 802.15.4 MAC with GTS Transmission for Heterogeneous Devices with Application to Wheelchair Body-Area Sensor Networks", IEEE Transactions on Information Technology in Biomedicine, vol. 15, no. 5, (2011), pp. 767-777. 


\section{Authors}

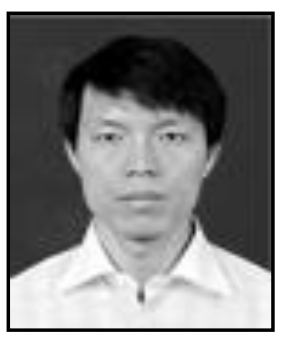

Fengjun Shang, he finished his Ph.D. degrees in Instrument Science and Technology at the College of Opto-electronic Engineering, Chongqing University, China, in 2005. Since then he works at the Institute of Computer Network Engineer in Chongqing University of Posts and Telecommunications, China. He was a visiting scholar in University of Wollongong, Australia, from November 2007 to November 2008. His research interests include sensor network, future internet, IOT, network optimization and Cloud Computing. 